\title{
Observation of periodic fluctuations in electron and ion temperatures at the low-latitude upper ionosphere by SROSS-C2 satellite
}

\author{
S. R. Prabhakaran Nayar ${ }^{1}$, L. T. Alexander ${ }^{1}$, V. N. Radhika $^{1}$, T. John ${ }^{2}$, P. Subrahmanyam ${ }^{2}$, P. Chopra ${ }^{2}$, M. Bahl ${ }^{2}$, \\ H. K. Maini ${ }^{2}$, V. Singh ${ }^{2}$, D. Singh ${ }^{2}$, and S. C. Garg ${ }^{2}$ \\ ${ }^{1}$ Department of Physics, University of Kerala, Trivandrum, 695 581, India \\ ${ }^{2}$ Radio and Atmospheric Science Division, National Physical Laboratory, New Delhi, 100 010, India
}

Received: 16 April 2003 - Revised: 10 September 2003 - Accepted: 21 October 2003 - Published: 8 April 2004

\begin{abstract}
The electron and ion temperature at the lowlatitude ionosphere exhibits a wide variety of fluctuations. Observations using SROSS-C2 satellite RPA payload during 1995-2000 provide an excellent opportunity to study the fluctuations in $T_{e}$ and $T_{i}$ in the upper ionosphere and to understand the dynamic processes existing at those altitudes. The spectral analysis of electron and ion temperatures using Fourier and wavelet techniques reveal the presence of quasiperiodicities, such as 14-day, 19-day, 27-day, 55-day, 154day, 180-day, 1-year and 1.3-year periods. The daytime and nighttime values of $T_{e}$ and $T_{i}$ exhibit different types of characteristic variations. The wavelet spectrum presented depicts the temporal evolution the power of each periodicity present in the daytime and nighttime values of $T_{e}$ and $T_{i}$. The time evolution of electron and ion temperatures are compared with that of $A_{p}$ and $10.7 \mathrm{~cm}$ solar radio flux, to infer the source of temperature fluctuations at the upper ionosphere.
\end{abstract}

Key words. Ionosphere (plasma temperature and density)

\section{Introduction}

The electron and ion temperatures at the upper ionosphere depends on both the solar and terrestrial conditions and is found to exhibit a variety of periodic variations found in solar and terrestrial parameters, such as diurnal, seasonal, latitudinal and solar activity associated variations (Brace et al., 1987; Oyama et al., 1996; Truhlik et al., 2000; Huba et al., 2000; Schunk and Nagy, 1978, 2000). The electron and ion temperatures have been observed using ground-based radars (McClure et al., 1973; Mahajan, 1977; Oliver, 1991; Otsuka et al., 1998), rockets (Oyama et al., 1991) and satellites (Brace et al., 1987). These measurements have shown that the electron temperature on a disturbed day is higher than that of the quiet day value (Rao, 1968; Rishbeth and Garriot,
1969). An empirical global model of electron temperature using Atmospheric Explorer-C (Brace and Theis, 1981) exhibited a broad region of low temperature in the nighttime equatorial ionosphere followed by a morning maximum, a decline to a daytime plateau and another increase at dusk. The electron and ion temperatures at the upper ionosphere show a sharp variation between day and night (Bhuyan et al., 2002; Nayar et al., 2003). In the day time $T_{e}$ in the plasmasphere rises sufficiently above theF-region temperature, resulting in a downward flow of heat. A complete understanding of the coupling between the F-region and plasmasphere can be obtained by studying the solar flares, neutral concentration, electron temperature and density $\left(T_{e}, N_{e}\right)$ and the flux of escaping photoelectrons. The electron temperature and density at the equatorial ionosphere have been modeled utilising the IRI model, computer simulation and Hinotori satellite measurements (Bhuyan et al., 2002; Oyama et al., 1996). During magnetic storms the $T_{e}$ increases due to the electric currents and charged particle precipitation associated with joule heating, traveling ionospheric disturbances and gravity waves. A number of local and non-local processes can affect the low-latitude thermosphere. Periodic fluctuations in the geomagnetic activity and ionospheric fluctuations result from the intrinsic state of the Sun, relative orientation of the Earth with respect to the Sun, evolution of solar surface features and interplanetary medium and processes at the Earth (Nayar et al., 2001, 2002). In this work, we have evaluated the electron and ion temperature of the low-latitude upper ionosphere using the RPA payload of the SROSS-C2 satellite during 1995 to 2000 . The $T_{e}$ and $T_{i}$ data has been separated into two time series representing daytime and nighttime and then used to investigate the periodicities present in them and their association with solar and terrestrial processes. The temporal evolution of these periodicities are also studied using the wavelet technique. 


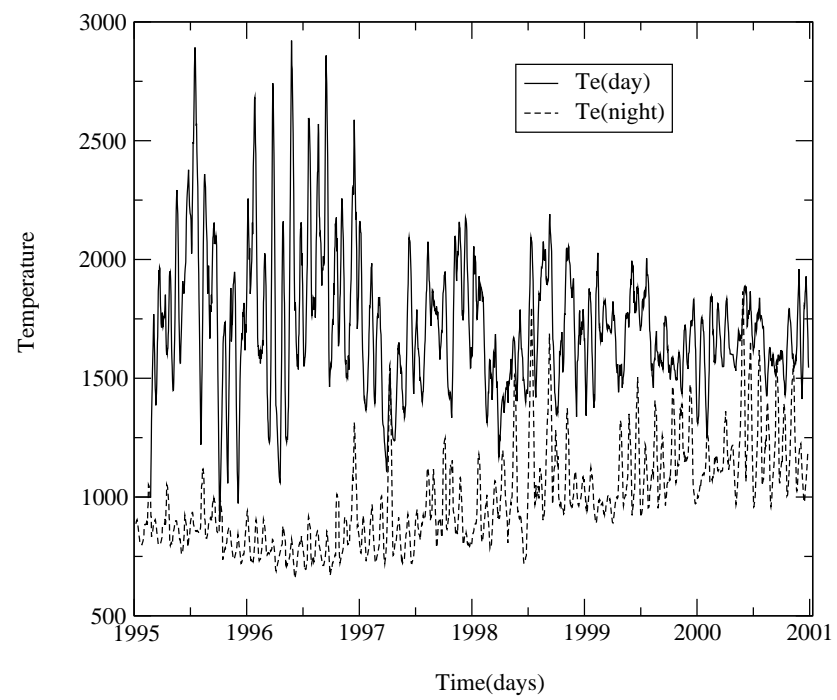

Fig. 1. Daytime (full line) and nighttime (dotted lines) average values of electron temperature during 1995-2000.

\section{Electron/ion temperature measurements by SROSS- C2 and method of analysis}

The SROSS-C2 satellite was launched from Shriharikota, India in May 1994. It is a spin-stabilized orbiting satellite placed in an elliptical orbit at $620 \times 420 \mathrm{~km}$ altitude, inclined at $46.3^{\circ}$ with equatorial plane. The satellite spins at about a rate of $5 \mathrm{rpm}$ and moves in cartwheel mode, keeping the spin axis perpendicular to the orbital plane. The electron and ion sensors have their axes perpendicular to the deck plane and parallel to the longitudinal axis of the satellite. The Retarding Potential Analyzer (RPA) payload on the SROSS-C2 satellite consists of an electron RPA, ion RPA and potential probe sensors and the associated electronics. The electron and ion RPAs are used for measuring the ionospheric electron and ion densities, their temperatures and other related parameters of plasma, providing a unique opportunity to study upper ionospheric phenomena. Normally the payload is operated for data collection only during overhead passes of the satellite over the ground telemetry stations. The satellite makes about 16 orbits per day, out of which, on average, two high elevation passes each during the daytime as well as the nighttime are visible from a single telemetry station. But due to operational reasons data collection on a regular basis is restricted to one day time and one nighttime orbit per day during overhead passes of the satellite over the ground station located at Bangalore $\left(12.5^{\circ} \mathrm{N}, 77.3^{\circ} \mathrm{E}\right)$. The satellite visibility varies from 7 to $12 \mathrm{~min}$., depending on the satellite elevation angle. For the data collection from the Bangalore ground station, latitudinal coverage extends from $5^{\circ} \mathrm{S}$ to $30^{\circ} \mathrm{N}$ and the longitude range can extend from $50^{\circ} \mathrm{E}$ to $100^{\circ} \mathrm{E}$.

The RPA probes make simultaneous measurements of electron and ion parameters along the orbital path of the satellite. The I-V characteristic curves obtained from the ion and electron RPA are used for retrieving these parameters us-

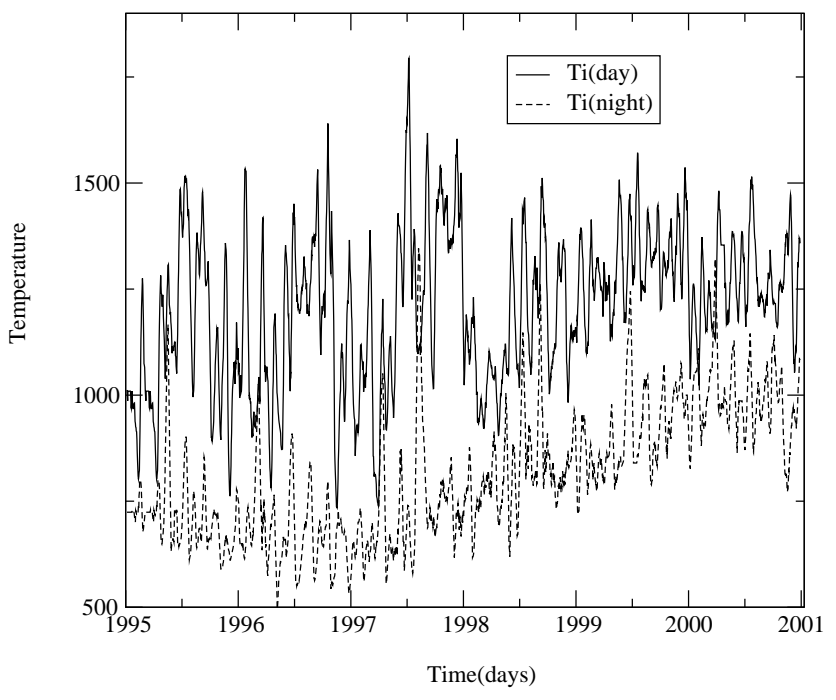

Fig. 2. Daytime (full line) and nighttime (dotted lines) average values of ion temperature during 1995-2000.

ing curve-fitting techniques. Data is collected from one daytime pass and one nighttime pass for RPA on a regular basis. The data is then separated into daytime and nighttime values by selecting the average $T_{e}$ and $T_{i}$ measurements made during each satellite visibility period. The daily values of daytime refers to the average of the measurements taken between 10:00 LT and 16:00 LT and the nighttime values refers to the measurements taken between 20:00 LT and 04:00 LT. The data gaps, when the satellite pass is outside the above mentioned time slots, are then filled using interpolation. For the evaluation of daytime and nighttime average values of $T_{e}$ and $T_{i}$, data from the entire range of latitude, longitude and altitude covered by SROSS-C2 satellite is used. Thus, these values are mixed with seasonal, altitudinal, latitudinal, longitudinal and solar cycle effects. From the daily daytime and nighttime average values a time series of $T_{e}$ and $T_{i}$ are generated. The electron and ion temperatures corresponding to daytime and nighttime are depicted in Figs. 1 and 2.This data is subjected to both Fourier and wavelet analysis to identify the presence of periods and their time evolution.

\section{Periodic variations in the ionosphere}

The ionosphere undergoes a marked diurnal variation as the Earth rotates into and out of the sunlight. The ionosphere exhibits strong seasonal and solar cycle variations because the main source of ionization and energy for the ionosphere is photoionization. In general, the Sun provides the driving force for nearly all geomagnetic and ionospheric fluctuations. Some of the geomagnetic and ionospheric fluctuations result from variations in the intrinsic state of the Sun. Solar features, the interplanetary medium and geomagnetic activity are found to exhibit a variety of periodicities (Donnelly and Puga, 1990; Murzula and Zeiger, 1996; Nayar et al., 2001, 2002). The periodic variations in the relative orientation of 
the Earth with respect to the Sun also introduces diurnal and seasonal periodicities in the ionospheric features. The study of periodicity in geomagnetic activity shows the presence of a variety of quasi-periodic variations (Fraser-Smith, 1972). Donnelly and Puga (1990) made extensive study of the solar 13.5-day periodicity at several wavelengths and found that the power of the 13.5-day periodicity is very dependent on the wavelength or source at the solar surface and does not behave as a sub-harmonic of the 27-day rotational period. It is the result of solar rotational modulation from two groups of active regions roughly $180^{\circ}$ apart in solar longitude. Most of these periodicities with periods more than 2 days are closely related changes associated with the Sun and are found to evolve with the phase of the solar cycle (Nayar et al., 2001, 2002). Periodicity around 155 days is of solar origin, observed in solar terrestrial parameters, and first observed in the occurrence frequency of solar flares (Rieger et al., 1984). Millstone Hill incoherent scatter radar detected quasi-periodic perturbations in electron density, ion velocity, electron temperature, and ion temperature at the F-region in the mid-latitude day-side ionosphere. This result shows that the mid-latitude ionospheric perturbations were well correlated with the IMF or solar wind pressure variations.

The planetary waves could also make a significant contribution to the dynamics at the ionospheric heights and they could be capable of causing significant day-to-day variability. Planetary waves with periods near 2, 5, 10, 16 days in the troposphere and stratosphere have been identified as a normal mode of oscillations of the atmosphere (Salby, 1984). The equatorial waves with periodicities in the range of 5-15 days, generated due to the latent heat release at the troposphere are reported to occur in the mesosphere regions. The 16day peak is interpreted in terms of leakage of a free Rossby wave from the stratosphere to the ionosphere (Forbes and Leveroni, 1992). Long period oscillations in the ionospherethermosphere system have their origin at the planetary waves (Vincent, 1990; Forbes and Leveroni, 1992). Propagating a gravity, tidal and planetary waves represent sources of energy and momentum responsible for upward vertical coupling from below in the atmosphere-ionosphere system (Lastovicka, 1997). The ionospheric $N_{e}$ found to exhibit a quasi18-day period which becomes modulated by the long period variations associated with the solar cycle. Spectral peaks obtained within the range of 10-20 days may be associated with the wave or 13.5-day periodicity, or with a combination of these effects. Periods between 20 days and 35 days are mainly due to variations in the solar flux and peaks with a period in the range of 10 to 20 days may be due to a combination of solar influences and planetary waves.

\section{Periodicities in SROSS-C2 RPA data of $T_{e}$ and $T_{i}$}

The electron and ion temperatures observed at the upper ionosphere using the RPA payload of the SROSS-C2 satellite exhibits a variety of variations, which include diurnal variations with morning overshoot, daytime plateau, evening

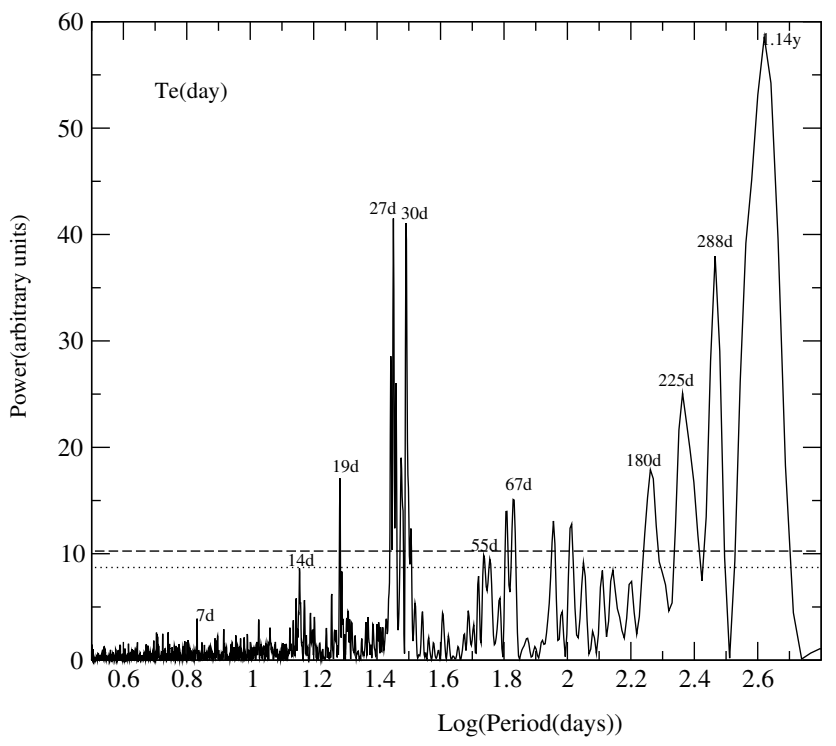

Fig. 3. Periodogram of daytime electron temperature. The numbers in the figure indicate the corresponding period in days. The dashed upper line is the upper limit of the $90 \%$ confidence level and the lower dotted line corresponds to the upper limit of $50 \%$ confidence level of the white noise spectrum.

enhancement, and nighttime minimum, 27-day solar rotation associated changes, seasonal variations and solar activity related variations. To study the periodicities present in these fluctuations, to probe the sources of the fluctuations in electron and ion temperatures, and to study the periodic variations in $T_{e}$ and $T_{i}$, the time series of the diurnal average values were subjected to both Fourier and Wavelet analysis. While the Fourier technique provides information about the average power of each period, the wavelet technique provides the time history of each period. The wavelet transform method gives good information on the time localization of each frequency component. The global wavelet spectrum, similar to the Fourier transform technique, provides information about the mean strength of each periodicity over the entire period of analysis.

\subsection{Fourier spectrum of $T_{e}$ and $T_{i}$}

The daily averaged values of $T_{e}$ and $T_{i}$ during daytime and nighttime are separated into two time series representing daytime and nighttime values over the period 1995-2000. These sets of data are subjected to Fourier analysis after de-trending and Lomb periodograms (Press et al., 2000) are obtained.

Figures 3 to 6 depicts the periodograms of electron and ion temperatures of the time series of daytime and nighttime averages in the period range a couple of days to 1.73 years.

Figure 3 depicts the spectrum of the electron temperature averaged over the nighttime observations at the upper ionosphere around $500 \mathrm{~km}$ altitude. The y-axis represents the spectral power in arbitrary units and the $\mathrm{x}$-axis depicts the $\log$ of period in days. The dashed upper line in Fig. 3 is the upper limit of the $90 \%$ confidence level and lower dotted line 


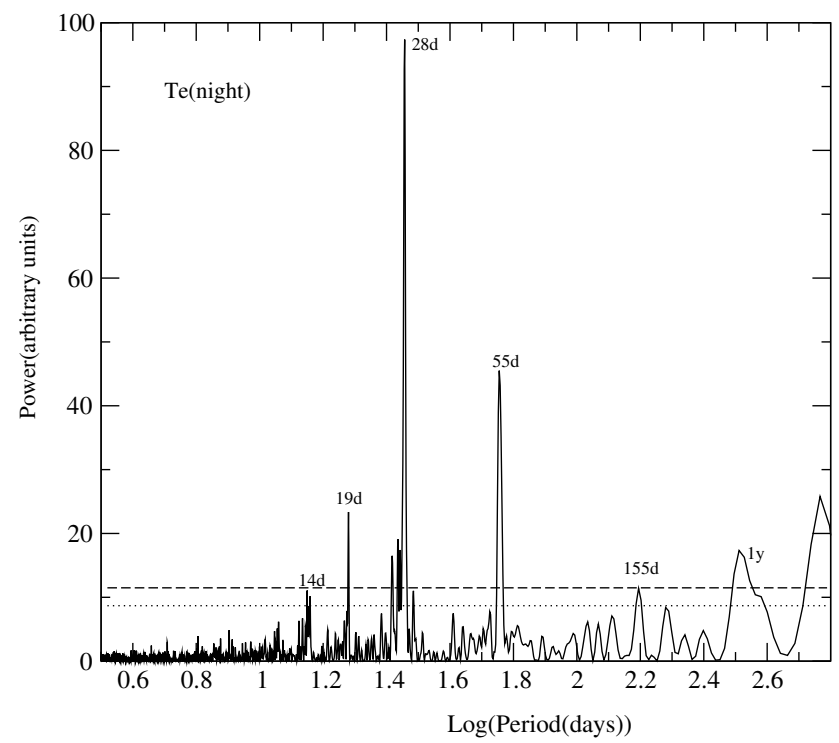

Fig. 4. Spectrum of nighttime electron temperature. The numbers in the figure indicate the corresponding period in days. Confidence levels same as in Fig. 1.

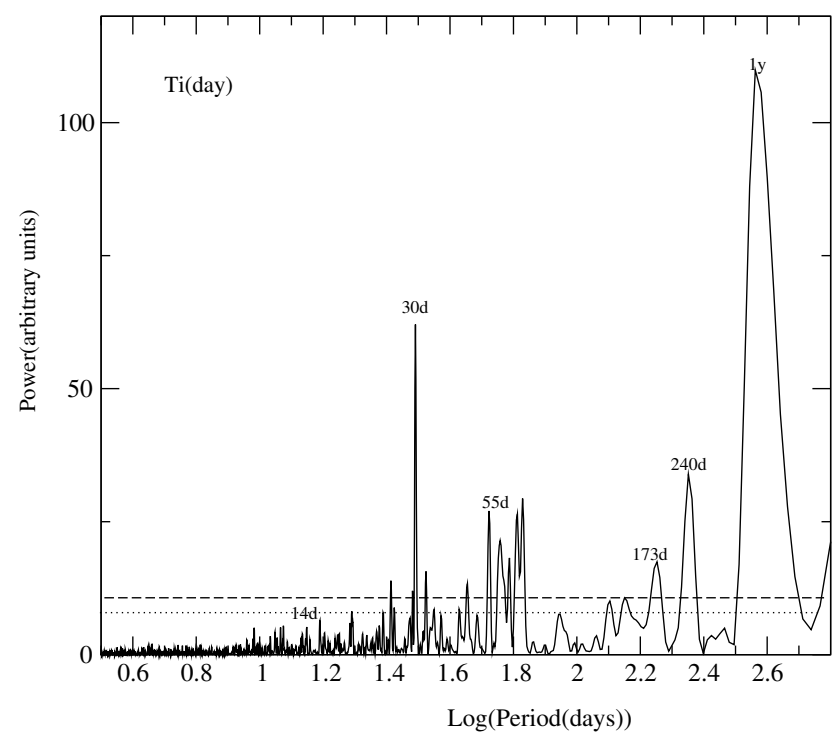

Fig. 5. Spectrum of daytime ion temperature. The numbers in the figure indicate the corresponding period in days. Confidence levels same as in Fig. 1.

corresponds to the upper limit of 50\% confidence level of the white noise spectrum deduced from the false-alarm probability of the null hypothesis (Press et al., 2000). In Fig. 3, the daytime values of $T_{e}$ depicts a variety of spectral peaks in the power corresponding to periods of around 14 days, 19 days, 27 days, 30 days, 55 days, 67 days, 180 days and about 1.1 years. The 27 -day period is associated with the solar rotation and the 55-day period is associated with the harmonic of the solar rotation period. The cause of the 30-day period is not yet known and it may be associated with the evolution of solar activity or with the planetary wave activity. The 14-day

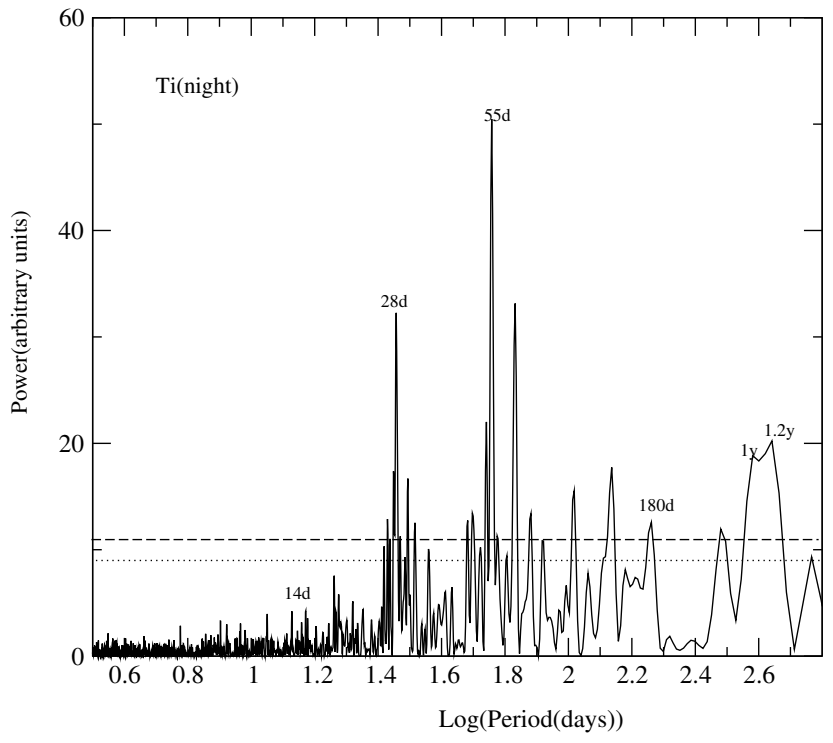

Fig. 6. Spectrum of nighttime ion temperature. The numbers in the figure indicate the corresponding period in days. Confidence levels same as in Fig. 1.

period is associated with the presence of active centres on the solar surface separated by $180^{\circ}$ in solar longitude. The source of the 19-day period is yet to be clearly understood. It can be either due to the solar activity variation or associated with the planetary wave activity. The 155 -day period is associated with the Rieger period (Rieger et al., 1984) observed in solar flares. The annual oscillation is also prominent in the daytime $T_{e}$ values.

Figure 4 depicts the spectrum of nighttime electron temperature fluctuations. Unlike the spectrum of daytime electron temperature, the nighttime electron temperature exhibits a period with very sharp peaks around 14 days, 19 days, 27 days, 55 days, 155 days and one year. Most of the spectral peaks are above the $90 \%$ confidence level. In Fig. 4, corresponding to the nighttime electron temperature, a smaller number of spectral lines are noticed compared to the spectrum of daytime $T_{e}$. In Fig. 4, the spectral peaks around 14 days, 19 days, 27 days, 55 days, 155 days, 1 year are clearly seen as prominent lines above the confidence level.

Figures 5 and 6 depict the spectrum of $T_{i}$ associated with daytime and nighttime values. The spectrum of $T_{i}$ also exhibits a few sharper spectral lines above the noise level, both during the daytime and the nighttime. While the 30-day period is prominent oscillation in $T_{i}$ during the daytime, the 28-day period is prominent in the nighttime data. The 55day, 180-day and 180-day periods are found in both the daytime and the nighttime $T_{i}$ data. The 1.3-year period is very prominent in the nighttime $T_{i}$ data. However, in all data sets the spectral lines are very broad around the 1 -year period.

\subsection{Wavelet spectrum}

Wavelet analysis is a tool for analyzing localized variations of power within a time series (Lau and Weng, 1995; 
Torrence and Campo, 1998; Nayar et al., 2002). By decomposing a time series into time-frequency space, wavelet transform has been used in a variety of fields and was found to be advantageous compared to the conventional tools for time series analysis. In the wavelet theory, we use wavelets which are special signals having amplitudes of definite duration and with specific frequency, which decay to zero in both positive and negative directions. Sets of wavelets are used to approximate a signal similar to the case of the Fourier series and each element in the wavelet is constructed from the same function called the mother wavelet, which is scaled and translated. Wavelet transform refers to the breaking down of the time series into many interrelated components. The scaling and translations are done simultaneously on the mother wavelet functions. The mother wavelet is the kernel of the wavelet transform from which a set of scaled and translated wavelets are created. The discrete wavelet transform is defined with respect to the mother wavelet $\psi$. After fixing the width of the wavelet, we can measure the projection of this wave packet on the time series data, i.e. by sliding the wavelet along the time series, we can determine the variation of projection amplitude with time. The choice of the wavelet function $\psi(\eta)$ at nondimensional time $\eta$ is made after considering its suitability for the application. In this analysis, we have used the Morlet wavelet, which is defined as the product of a complex exponential wave and a Gaussian envelope. The value of wavelet transform can be calculated for various values of the scale which is usually taken as the multiple of the lowest frequency possible. A two-dimensional plot of the variability can be constructed by plotting the wavelet amplitude and phase. For the present study we have used the Morlet wavelet defined as $\psi_{0}(\eta)=\pi^{-1 / 4} e^{i \omega_{0} \eta} e^{-\eta^{2} / 2}$, where $\omega_{0}$ is the wave number taken to be 6 . In the case of the Morlet wavelet for $\omega_{0}=6$, the Fourier period is 1.03 times the wavelet period (Torrence and Campo, 1998). If the wavelet power spectrum is significantly above the mean power spectrum, then it can be assumed to be a true factor with a certain percent confidence. For the present analysis we have used the $T_{e}$ and $T_{i}$ data of the upper ionosphere observed by the RPA payload of the SROSS-C2 satellite. The data of $T_{e}$ and $T_{i}$ is available during 1995-2000 as daily average values. From these parameters, both global spectra and scalograms are obtained to study the periods in them and to understand the evolution of periodicities with time. The global spectra is similar to the Fourier spectra, giving the average strength of each period during the entire range of observation period. The scalogram provides information about the evolution of the power associated with each period. In this work, we have restricted our analysis to periods from a few days to 2 years.

\subsubsection{Global wavelet spectra of $T_{e}$ and $T_{i}$}

In this section, the presence of periodicities in electron and ion temperatures in the upper ionosphere have been analyzed by the wavelet technique by looking at their temporal average behaviour using global wavelet spectrum, which is the plot of the mean wavelet power against the wavelet period.

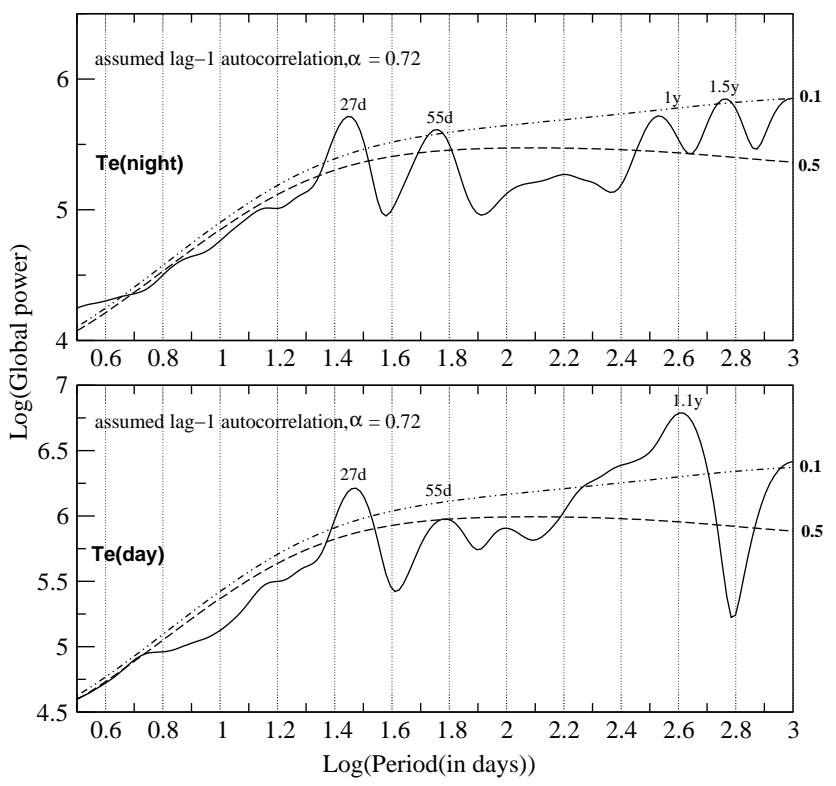

Fig. 7. Global wavelet spectra of average values of electron temperature during daytime and that of nighttime. The dotted line indicates the $90 \%$ confidence level and the dashed line indicates the $50 \%$ confidence level of the red noise background spectrum.

In this case, since we use the Morlet wavelet, the wavelet scale/period is nearly equal to the Fourier period. So the global spectrum is similar to the Fourier spectrum with the wavelet period and the Fourier period differing depending on the selection of the wavelet. In Figs. 7 and 8, the upper dashed lines indicates the $90 \%$ confidence level and the lower dashed lines indicate the $50 \%$ confidence level of the red noise background spectrum (Torrence and Campo, 1998). Most prominent periods present in the global wavelet spectra of electron and ion temperature are around 27 days, 55 days, 154 days and 1 year. The global wavelet spectra of $T_{e}$ (day), $T_{e}$ (night), $T_{i}$ (day) and $T_{i}$ (night) in the period range of 1day (0.05) to 3-year (3.2) are depicted in Figs. 7 and 8. The upper curve in Fig. 7 depicts the global wavelet spectrum of nighttime $T_{e}$ and the lower curve depicts the daytime $T_{e}$. As in the case of the Fourier spectra, the 27-day and 55-day periods are prominent in the $T_{e}$ nighttime data. Similarly for $T_{e}$ at the daytime (Fig. 7 lower) 27-day and 1-year periods are prominent and seen above the noise level.

The upper graph in Fig. 8 indicates the global wavelet spectra of nighttime $T_{i}$ data and the lower portion depicts the global spectrum of $T_{i}$ during the daytime. In the case of $T_{i}$ data, both during the daytime and the nighttime, the 27day, 55-day and 1-year wavelet periods are seen above the $90 \%$ confidence level. Since the global wavelet spectra is the time average of the wavelet power during different epochs, the spectral peaks are broader and many spectral peaks vanished below the confidence level. 


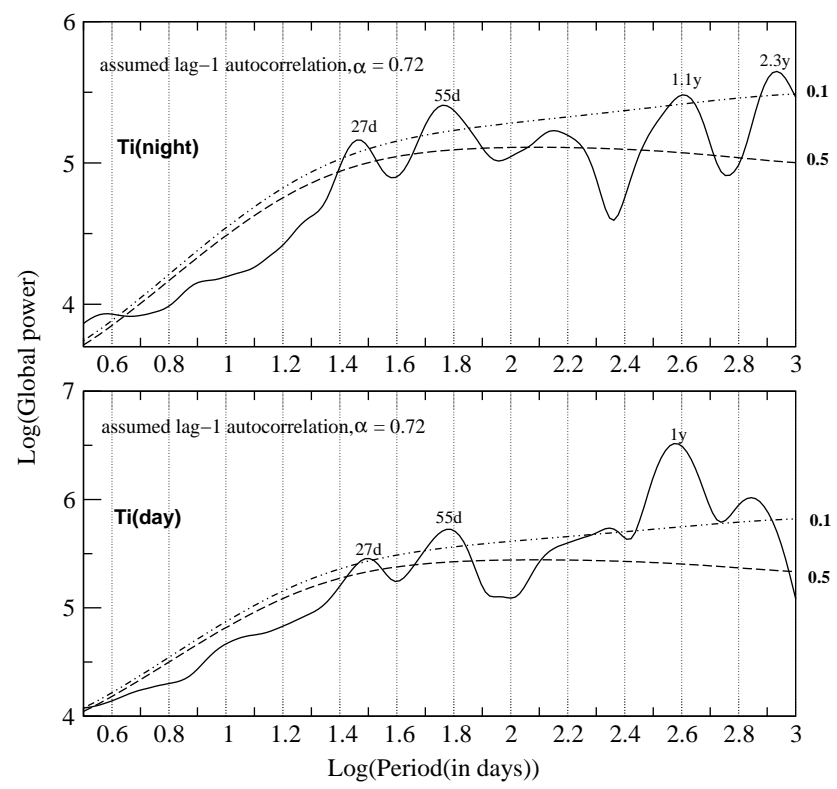

Fig. 8. Global wavelet spectra of average values of ion temperature during daytime and that of nighttime. Confidence levels same as described in Fig. 7.

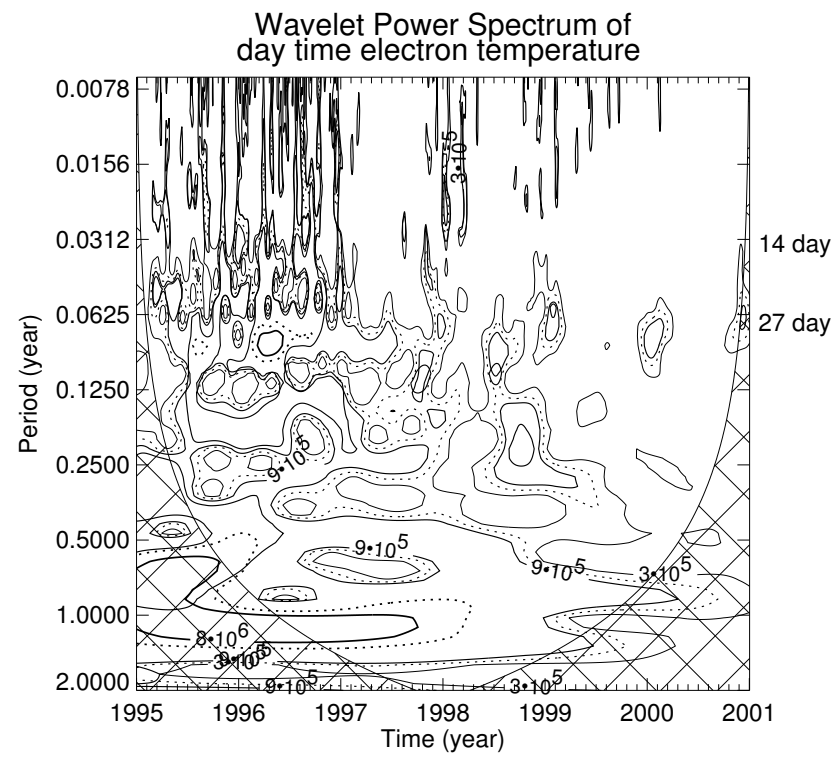

Fig. 9. Wavelet spectra of electron temperature during the daytime. The wavelet scale is marked in years along the y-axis. The power corresponding to each contour is marked in figures. The mesh indicates the regions where the edge effect becomes important.

\subsubsection{Wavelet spectrum of $T_{e}$ and $T_{i}$}

Figures 9 to 12 depict the wavelet spectra of $T_{e}$ and $T_{i}$ during daytime and nighttime. The wavelet spectrum is a periodogram depicting the evolution of wavelet power with time. Since we use the Morlet wavelet for the present analysis, the wavelet period is nearly the same as that of the Fourier period in Figs. 9 to 12. The wavelet power is not evenly distributed

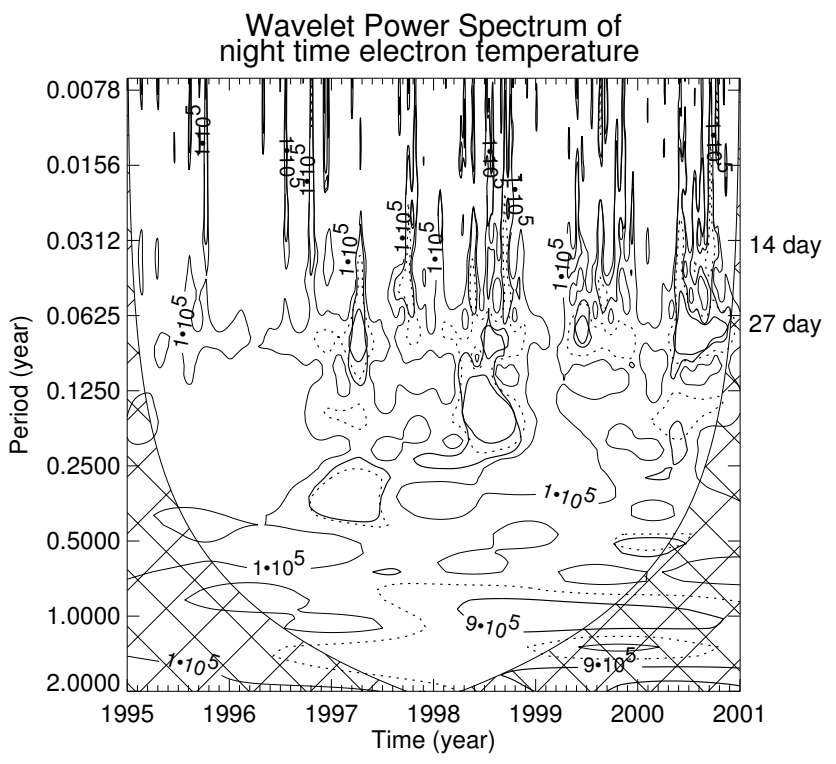

Fig. 10. Wavelet spectra of electron temperature during the nighttime. Other details same as Fig. 1.

in the periodogram. The power is concentrated at certain period bands and is found to evolve with time. Since the data used is not cyclic, to minimise errors at the beginning and end of the wavelet spectrum, the time series of $T_{e}$ and $T_{i}$ are padded with zeroes to bring the data length to a power of two. This introduces a decrease in amplitude at the end points as one goes to larger scales. The region indicated by the mesh indicates the region of the spectrum in which edge effects become important. Figure 9 depicts the time history of the wavelet power of the fluctuations in the daytime $T_{e}$ during 1995-2000. It is noticed that during 1995-1997, most of the periods exhibit larger wavelet power compared to later years. The 14-day period has larger power around 1996 and the 19day period has larger power around 1995-1996. The 27-day, 55-day, 180-day and 1 to 1.3-year periods are prominent during the three years 1995-1997.

Figure 10 depicts the time evolution of the wavelet power of the average nighttime electron temperature. Unlike the daytime $T_{e}$ data, the nighttime $T_{e}$ data exhibit larger wavelet power during the period 1997-2000 for the 14-day, 19-day, 27-day, 180-day and 1-year periods. The wavelet power of short period oscillations around 14 days, 19 days and 27 days show periodic variations in their strength during the period. During 1998, the wavelet power is found to be stronger below the 100-day period.

Figure 11 depicts the time history of the wavelet power of ion temperature during the day time. The 27- and 55day periods are stronger in the ion temperature with a peak wavelet power around 1996-1997. The periods around 155 days is prominent during 1997. The 180 days and 1 year also exhibit maximum power around 1997. During 1997, the wavelet power enhances around 1.3-year period.

Figure 12 depicts the temporal evolution of the wavelet power of ion temperature at nighttime. The wavelet spectra 


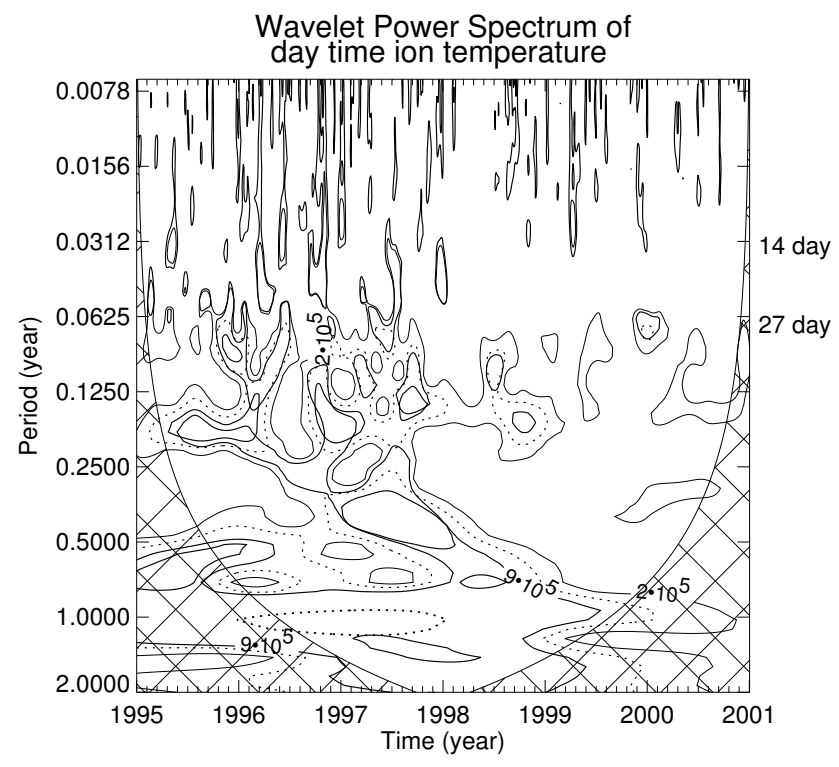

Fig. 11. Wavelet spectra of ion temperature during the daytime. Other details same as in Fig. 1.

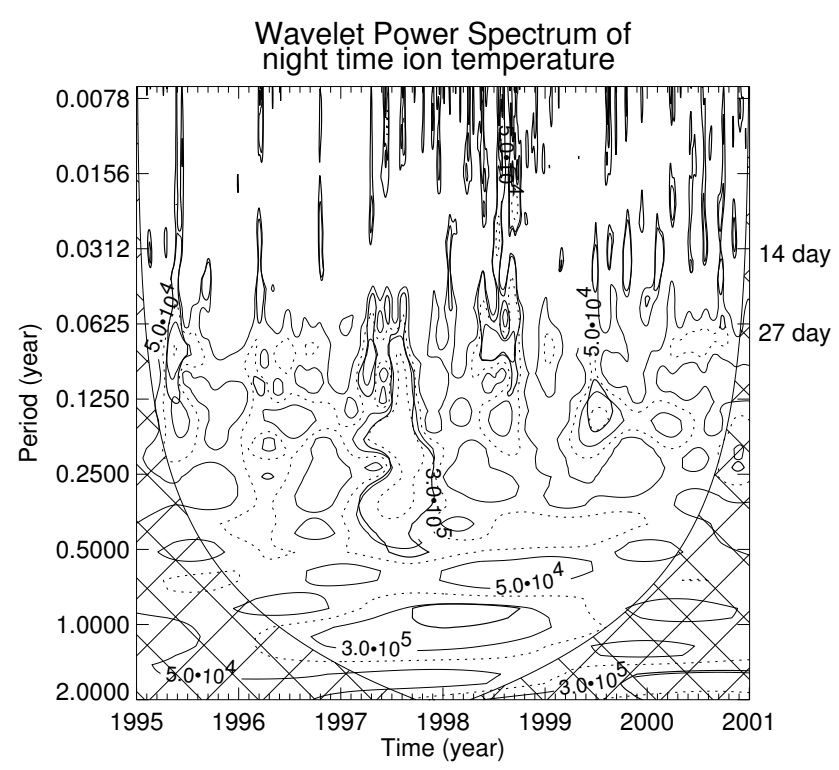

Fig. 12. Wavelet spectra of ion temperature during the nighttime. Other details same as in Fig. 1.

of $T_{i}$ at night exhibits peak values around 1995, 1997, 1998. Similarly the wavelet power of the 55-day period also exhibit an intermittent increase in wavelet power. The 180-day period is found to be prominent during 1997-1999 and the 1-1.3 years period prominent during 1998-1999. A strong enhancement in the wavelet power is noticed in a narrow time period around 1997, in the period range 27 days to 180 days.

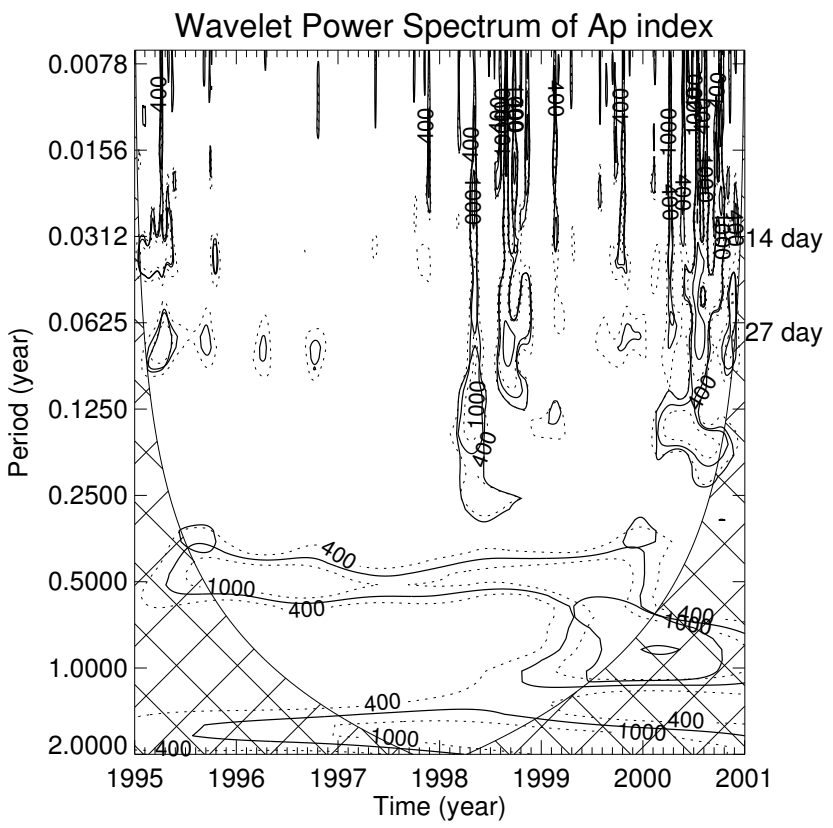

Fig. 13. Wavelet spectra of $A_{p}$ during 1995-2000. Other details are same as in Fig. 1.

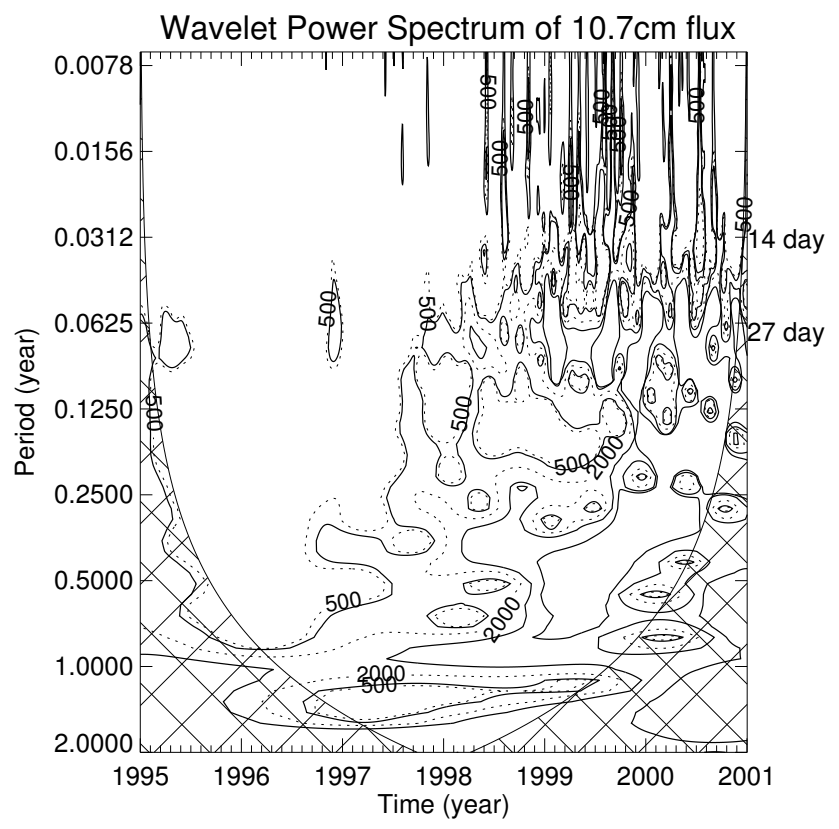

Fig. 14. Wavelet spectra of solar radio flux during 1995-2000. Other details are same as in Fig. 1.

4.3 Cross wavelet spectrum of $A_{p}$ and F10.7 flux with electron and ion temperatures

To identify the cause of periodic fluctuations in the daytime and nighttime $T_{e}$ and $T_{i}$ and their association with solar activity, we have studied the wavelet spectra of the geomagnetic activity index $A_{p}$ and solar radio flux F10.7. The geomagnetic activity index $A_{p}$ is directly related to the solar wind 


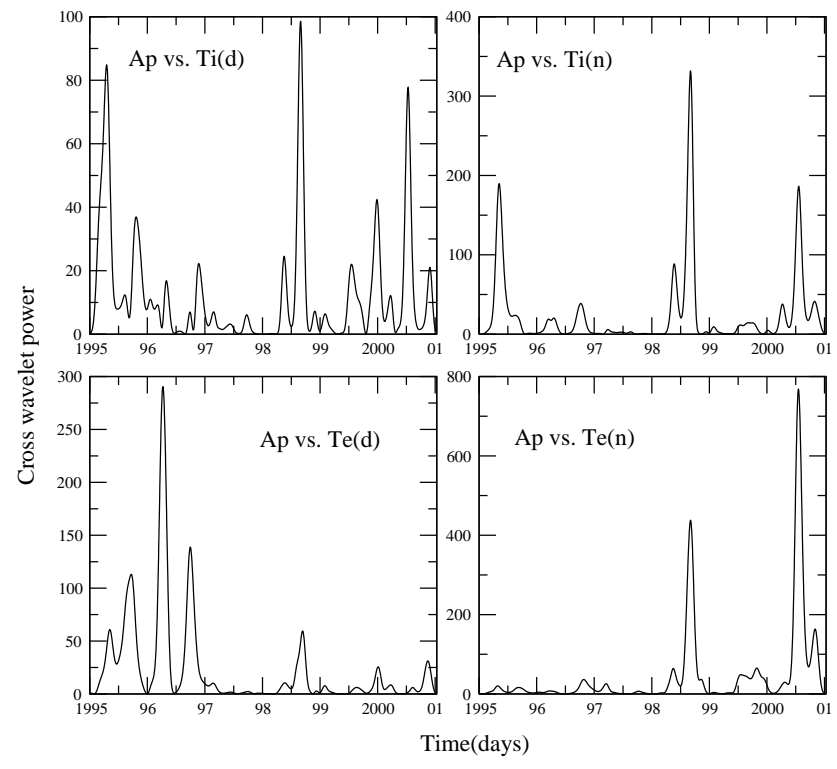

Fig. 15. Time evolution of cross wavelet spectrum between $A_{p}$ and electron and ion temperatures for the 27-day periodicity during 1995-2000.

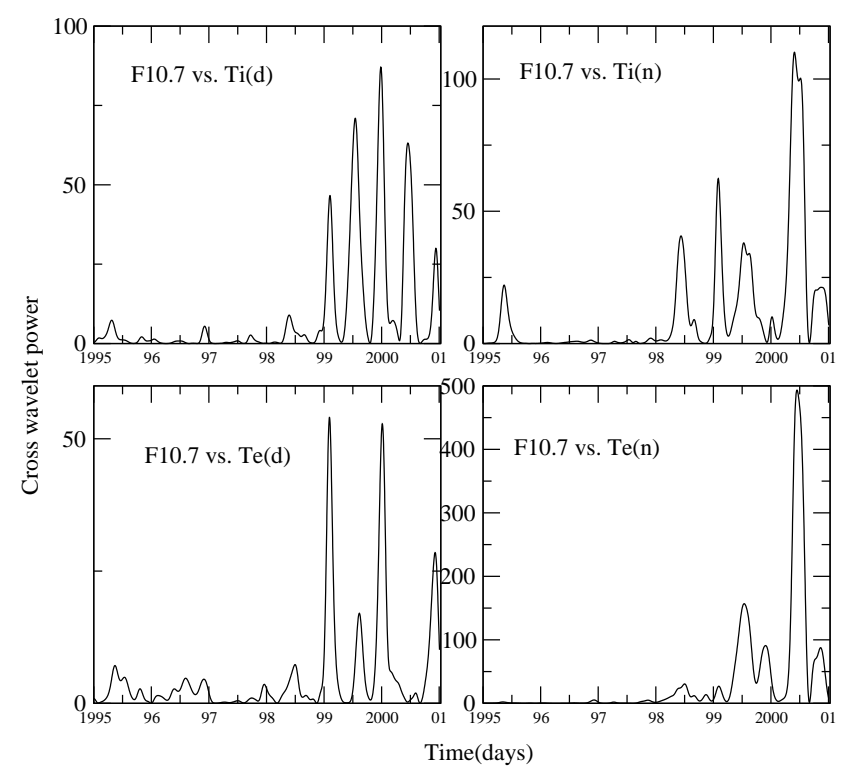

Fig. 16. Time evolution of cross wavelet spectrum between solar radio flux and electron and ion temperatures for the 27-day periodicity during 1995-2000.

velocity, and the solar radio flux is an indicator of solar activity and the sunspot number. Figures 13 and 14 depict the time evolution of the wavelet power for different periods in the $A_{p}$ index and F10.7. The $A_{p}$ index exhibits larger wavelet power associated with a semiannual period during 1995-1999 and around a 1-year period during 2000. Due to poor resolution, the 155-day period is merged within the semiannual period band and the 1.3-year period is merged within the 1-year band. The 14-day and 27-day periods in $A_{p}$ are prominent during 1995-1996. During 1998 and 2000 most of the short period oscillations in the period band less than 100 days exhibit larger wavelet power. Compared to the $A_{p}$ index, the F10.7 index depicts larger wavelet power during 1998-2000 and the wavelet power is found to be highly evolving during this period without the dominance of any particular period.

For a quantitative evaluation of the relation between the wavelet powers of $T_{e}$ and $T_{i}$ with $A_{p}$ index and F10.7 index, the cross wavelet power is evaluated for a typical period of 27 days. Figure 15 depicts the cross wavelet power between $A_{p}$ index and the electron temperatures at daytime (Te(d)) and nighttime $(\operatorname{Te}(\mathrm{n}))$ and ion temperature at daytime $(\operatorname{Ti}(\mathrm{d}))$ and nighttime (Ti(n)) during 1995-2000, expressed in days. The relation between the $A_{p}$ index and the ionospheric temperatures at the 27-day period is found to be stronger during the solar minimum years 1995-1996. During 1998 a prominent enhancement in the cross wavelet power is noticed. In the case of the cross wavelet power between the F10.7 index and electron and ion temperatures during 1995-2000 is as depicted in Fig. 16. It is noticed that in general the cross wavelet spectrum is stronger during 1998-2000, indicating that the wavelet power of the 27-day wavelet scale in $T_{e}, T_{i}$ and F10.7 are well correlated during 1998-2000.

\section{Discussion of the results}

In this work, the periodicities present in the electron and ion temperatures at the upper ionosphere around $500 \mathrm{~km}$ altitude have been studied. The electron and ion temperatures at the low-latitude topside ionosphere are evaluated from the observation of I-V characteristic curves obtained using RPA payload on board the SROSS-C2 satellite and are separated into daytime and nighttime average values for each day and presented in Figs. 1 and 2. The characteristics of these fluctuations in $T_{e}$ and $T_{i}$ studied using Fourier and wavelet techniques provide complementing information. While the Fourier spectra presented in Figs. 3-6 depicts the Fourier power corresponding to periods of 14 days, 19 days, 27 days, 55 days, 155 days, 180 days, 1 year and 1.3 years. While the Fourier spectrum gives information about the power for the entire period, the wavelet analysis (Figs. 7-12) provides information about their time history during 1995-2000. The presence of these periods in electron and ion temperatures, along with other solar and terrestrial parameters (Nayar et al., 2002), give an indication of the sources of heating at the upper ionosphere. The source of the 14-day periodicity is the presence of two active centres on the solar surface separated by $180^{\circ}$ solar longitude. The 27 -day period is associated with the solar rotation and the 55-day period is associated with its harmonics. The 154-day period is called the Rieger period associated with the recurrence of solar flares. The 1.3-year period associated with the evolution of solar rotation is found in many solar and interplanetary parameters. These periods affect both the solar radiation output and the characteristics of the outflow of solar wind and interplanetary magnetic field. The annual and semiannual periods are associated with the solar terrestrial relation found in many 
terrestrial activity indices. The wavelet spectra of $A_{p}$ and F10.7 indices evaluated for the period 1995-2000 (Figs. 13 and 14) enables one to compare similar features in these indices and the ionospheric plasma temperatures. The cross wavelet spectrum presented in Figs. 15 and 16 indicates the temporal evolution of the relation between them.

The ionospheric variations are influenced by changes in solar ionizing radiations, variations in solar wind plasma parameters and other variations due to vertical transport of planetary wave energy generated at the lower atmosphere. The electron and ion temperatures at the upper ionosphere are also affected by the electric field variations and the related density changes. The study of the characteristics of the Fourier and wavelet spectrum help us to infer the prominent processes associated with the heating of the upper ionosphere. Most of the periods present in $T_{e}$ and $T_{i}$ are associated both with the solar wind and solar radiation, and are associated with active centres at the solar surface. Though some of the periods have their Fourier power below the significant level, the wavelet spectrum indicates their prominence during certain epochs. The study of the similarities of the time evolution of the wavelet power of $A_{p}$ and F10.7 indices with $T_{e}$ and $T_{i}$, and the cross wavelet spectrum indicate that the plasma temperature at the upper ionosphere is controlled by solar wind around the solar minimum and by both the solar radiation and solar wind around solar maximum. These results suggests that the processes associated with the heating of ionospheric plasma by the solar radiation and the solar wind depends on the phase of the solar cycle and the conditions of the solar radiation, interplanetary medium and magnetosphere.

The periodicity around 19 days observed in the $T_{e}$ and $T_{i}$ spectrum can be utilised to study the possible contribution of planetary waves generated at the lower atmosphere on the upper ionosphere temperature. In the spectrum of electron and ion temperatures, the 19-day period has significant spectral power. The wavelet spectrum of the $A_{p}$ index and F10.7 does not exhibit this periodicity. Dynamical coupling through various types of waves generated at the lower altitudes play an important role in the energy transport between lower and upper atmosphere. These waves grow in amplitude as they propagate to higher altitudes and carry significant amounts of energy and momentum to be able to affect the thermospheric heights (Krishna Murthy, 1998). Even though planetary waves are unable to propagate directly to the upper ionosphere, planetary wave type oscillations have been observed at these heights. The 18-day quasi-period exists and persists in the ionospheric electron density variations with mean period around 18-19 days (Altadill, 1996). The 18-day quasi-periodic oscillation is also present in solar parameters Rz, F10.7, with a mean period around 19-20 days (Wilson, 1982). These results indicate that the period around 19 days can have a contribution from solar and terrestrial activities. So, the quasi-periodic oscillations present in the ionospheric electron and ion temperatures can be used to infer the source of heating at the upper ionosphere and the processes existing at those altitudes.
Acknowledgements. The authors thank the Indian Space Research Organisation for supporting part of this work under RESPOND scheme. One of the authors (LTA) acknowledge the financial support by University Grants Commission and (VNR) for the award of fellowship by CSIR.

Topical Editor M. Lester thanks a referee for the help in evaluating this paper.

\section{References}

Altadill, D.: On the 18 day quasi-periodic oscillation in the ionosphere, Ann. Geophys., 20, 807-815, 1996.

Bhuyan, P. K., Chamua, M., Subrahmanyam, P., and Garg, S. C.: Diurnal, seasonal and latitudinal variations of electron temperature measured by SROSS C2 satellite at $500 \mathrm{~km}$ altitude and comparison with IRI, Ann. Geophys., 20, 807-815, 2002.

Brace, L. H. and Theis, R. F.: Global empirical models of ionospheric electron temperature in the upper F-region and plasmasphere based on in situ measurements from the atmosphere Explorer-C ,ISIS-1 and ISIS-2 satellites, J. Atmos. Terr. Phys., 43, 1317-1343, 1981.

Brace, L. H., Theis, R. F., and Hoegy, W. R.: Ionospheric electron temperature at solar maximum, Adv. Space Res., 7, 99-106, 1987.

Donnelly, R. F. and Puga, L. C.: Thirteen day periodicity and the centre-to-limb dependence of UV, EUV and X-ray emission of solar activity, Solar Phys., 130, 369-390, 1990.

Forbes, J. M. and Leveroni, S.: Quasi 16-day oscillation in the ionosphere, Geophys. Res. Lett., 19, 981-984, 1992.

Fraser-Smith, A. C.: Spectrum of geomagnetic activity index $A_{p}$, J. Geophys. Res., 77, 4209-4220, 1972.

Huba, J. D., Joyce, G., and Fedder, J. A.: Sami2 is another model of the ionosphere(SAMI2): A new low-latitude ionosphere model, J. Geophys. Res. , 105, 23 035-23 053, 2000.

Krishna Murthy, B. V.: Middle atmosphere upper atmosphere coupling, Proc. Indian National Sci. Acad., 64, 303-313, 1998.

Lastovicka, J.: Observations of tides and planetary waves in the atmosphere ionosphere system, Adv. Space Res., 20, 1209-1222, 1997.

Lau, K. M. and H. Y. Weng: Climate signal detection using wavelet transform: How to make time series sing, Bull. Amer. Met. Soc., 76, 2391-2402, 1995.

Mahajan, K. K.: Models of electron temperature in the ionospheric F-region using electron density height profiles, J. Atmos. Terr. Phys., 39, 637-639, 1977.

McClure, J. P., Hanson, W. B., Nagy, A. F., Cilrone, R. J., Brace, L. H., Baron, M., Bauer, P., Carlson, H. C., Evans, J. V., Taylon, G. N., Woodman, R. F.: Comparison of Te and Tifrom OGO-6 and from various incoherent scatter radars, J. Geophys. Res., 78, 197-205, 1973.

Mursula, K. and Zieger, B.: The 13.5 day periodicity in the Sun, solar wind, and geomagnetic activity: The last three solar cycle, J. Geophys. Res., 101, 77-90, 1996.

Nayar, S. R. P., Sanalkumaran Nair, V., Radhika, V. N. and Revathy, K.: Short period features of the interplanetary plasma and their evolution, Solar Phys., 201, 405-417, 2001.

Nayar, S. R. P., Radhika, V.N., Ramadas, V., and Revathy, K.: Wavelet analysis of periodicities in the interplanetary medium, Solar Phys., 208, 359-373, 2002.

Nayar, S. R. P., Alexander, L. T., John, T., Subrahmanyam, P., Chopra P., Bahl, M., Maini, H. K., Singh, V., Singh, D., and 
Garg, S. C.: Study of the evolution of Te and Ti at low-latitude upper ionosphere using SROSS-C2 RPA observations, J. Atmos. Solar Terr. Phys.(communicated 2003).

Oliver, W. M.: Measurement of ionospheric and thermospheric temperatures and densities with the middle and upper atmosphere, J. Geophys. Res., 96, 827-838, 1991.

Otsuka, Y., Kawamura, S., Balan, N., Fukao, S., and Bailey, G. J.: Plasma temperature variation in the ionosphere over the middle and upper atmosphere radar, J. Geophys. Res., 103, $20705-$ 20713, 1998.

Oyama, K. I.: Electron temperature measurements carried out by JAPANESE scientific satellites, Adv. Space Res., 11, 149-158, 1991.

Oyama, K. I., Watanabe, S., Su, Y., Takahashi, T., and Hirao, K.: Season, local time and longitude variations of electron temperature at the height of $600 \mathrm{~km}$ in the low-latitude region, Adv. Space Res., 18, 269-278, 1996.

Press, W. H., Teukilsky, S. A., Vetterling, W. T., and Flannery, B. P.: Numerical recipes in C: The art of scientific computing, Cambridge University Press, New Delhi, 2000.

Rao, P. B.: Electron concentrations and electron and ion temperatures in theF-region for magnetically quiet and disturbed conditions, J. Geophys. Res. , 73, 1661-1678, 1968.
Rieger, E., Share, G. H., Forrest, D. J., Kanbach, G., Reppin, C., Chupp, E. L.: A 154-day periodicity in the occurrence of hard solar flares, Nature , 312, 623-625, 1984.

Rishbeth, H. and Garriot, O. K.: Introduction to the ionospheric physics, Academic Press, New York, 1969.

Salby, M. L.: Survey of planetary scale travelling waves: The state of theory and observations, Rev. Geophys. Space Phys. 22, 237244, 1984.

Schunk, R. W. and Nagy, A. F.: Electron temperatures of the Fregion of the ionosphere-Theory and observations, Rev. Geophys. Space Phys., 96, 355-399, 1978.

Schunk, R. W. and Nagy, A. F.: Ionospheres: Physics, plasma physics and chemistry, Cambridge University Press, Cambridge, 2000.

Torrence, C. and Compo, G. P.: A practical guide to wavelet analysis, Bull. Amer. Met. Soc., 79, 61-78, 1998.

Truhlik, V., Triskova, L., Smilauer, J., and Afonin, V. V.: Global empirical model of the electron temperature in the outer ionosphere for period of high solar activity based on data of three inter-Cosmos satellites, Adv. Space Res., 25, 163-169, 2000.

Vincent, R. A.: Planetary and gravity waves in the mesosphere and lower thermosphere, Adv. Space Res., 10, 93-101, 1990.

Wilson, R. C.: Solar irradiance variations and solar activity Journal of Geophysical research, J. Geophys. Res., 87, 4319-4326, 1982. 